\title{
The CXCL12/CXCR4 pathway or the autocrine proliferative loop of the glioblastoma stem cells
}

\author{
Bernard Rogister ${ }^{1,2}$ \\ ${ }^{1}$ Laboratory of Nervous System Disorders and Therapy, GIGA-Neurosciences Research Center, University of Liège, Liège, Belgium; ${ }^{2}$ Department \\ of Neurology, CHU and University of Liège, Liège, Belgium \\ Correspondence to: Bernard Rogister, MD, PhD. Laboratory of Nervous System Disorders and Therapy, GIGA-Neurosciences Research Center, \\ University of Liège, Avenue Hippocrate, 15, 4000 Liège, Belgium. Email: Bernard.Rogister@ulg.ac.be. \\ Provenance: This is a Guest Editoral commissioned by the Section Editor Ning Huang (Department of Neurosurgery, The Second Affiliated Hospital \\ of Chongqing Medical University, Chongqing, China). \\ Comment on: Calinescu AA, Yadav VN, Carballo E, et al. Survival and Proliferation of Neural Progenitor-Derived Glioblastomas Under Hypoxic \\ Stress is Controlled by a CXCL12/CXCR4 Autocrine-Positive Feedback Mechanism. Clin Cancer Res 2017;23:1250-62.
}

Submitted Feb 01, 2017. Accepted for publication Feb 08, 2017.

doi: $10.21037 /$ tcr.2017.03.47

View this article at: http://dx.doi.org/10.21037/tcr.2017.03.47

Despite multimodal therapy (the classical Stupp's protocol) including surgical resection (as large as possible), radiotherapy and chemotherapy, glioblastoma (GBM) remain a burden as the global survival rate at two years reaches barely $9 \%$ of patients (1). This situation is mainly a consequence of a systematic recurrence and this recurrence is itself a consequence of various causes, acting alone or synergistically: heterogeneous nature of the disease (2), the presence of the brain-blood barrier which impedes the potentially active drugs to get into the brain (3), the lack of targeted chemotherapeutic molecules and the persistence of GBM-initiating or stem cells (GSC) (4).

The recent study of Calinescu et al. (5) specifically address the question of GSC as those cells have been demonstrated to be resistant to chemo- and radiotherapy $(6,7)$ and responsible for promoting neo-angiogenesis in tumors (8). Indeed, the authors induced brain tumors in a model recently described and based on a plasmid which allow a knock-in of immature brain cells using a Sleeping Beauty transposase (9). The inserted genes are the simian virus 40 large $T$ antigen (SV4O- $L g T$ ) and a constitutively active human NRAS oncogene (NRAS). The plasmid construct was injected in the right lateral ventricle of postnatal day 1 mice expressing the dsRed fluorescent protein under the control of the CXCL12 promoter. Ten days after the plasmid injection, one can observe clusters of cells in the sub-ventricular zone (SVZ) expressing the SV40-LgT, but also Nestin and Olig2, two markers of cell immaturity in the brain. These cell clusters are near capillary where endothelial cells express the chemokine CXCL12. Nineteen days after the plasmid injection, the tumors can be observed, some present in the SVZ and some in the brain parenchyma. Those tumors are surrounded by reactive astrocytes expressing high levels of GFAP. Those tumors express Nestin and Olig2 but also exhibit several GBM hallmarks (multinucleated cells, numerous mitosis, vascular proliferation, pseudopalisading necrosis). Moreover, there is a lack of an efficient brain-blood barrier as intravascular dextrans are able to diffuse into the tumor.

Those tumors have been put in cultures and Calinescu et al. demonstrated that some tumoral cells grown as spheres, express CD133 and are able to generate tumors when transplanted back in naïve mice. All these features are specificities of GSG. Therefore, the authors compare the transcriptome of those cells (named M7) to two other GBM established mouse cell lines (GL26 and GL26A1, the latter is overexpressing NRAS). They found a differential expression for 5866 probe sets, in which several chemokines: CCL2, CXCL1, CCL7 and CXCL12. They focused to CXCL12 for two reasons: (I) they wanted to characterize the molecular environment of GSC that is responsible for the set-up of a niche for these tumor stem cells; and (II) CXCL12 has been previously shown to regulate neural and hematopoietic niches, acting through its CXCR4 receptor (10). 
Indeed, it appears that, at the phylogenetical point of view, the CXCL12/CXCR4 axis is very old, existing before the appearance of an adaptive immune response, with ancestral role in the nervous system (11).

Calinescu et al. quantified and characterized the expression of both CXCL12 and CXCR4 in their GBM model. They showed that the cytokine is expressed at high levels in the GBM induced by the transforming plasmid but also in the surrounding brain tissue. CXCR4, the CXCL12 receptor, is expressed at high level by tumoral cells, by immune infiltrating cells but not by normal brain cells. Finally, CXCR7, the other and less known CXCL12 receptor, is barely detectable in the system. CXCL12 and CXCR4 are also expressed by cultivated tumoral cells and AMD3100 or Plerixafor, the classical CXCR4-inhibitor, stimulates in vitro the early apoptosis (modestly) and inhibits (more strongly) the cell proliferation. Indeed, cell cycle analysis revealed that AMD3100 maintains cells in G2/M phases and decreases the number of cells in S-phase. This could be a consequence of a decrease of expression of early G1 cyclins and CDKs like cyclin D1, CDK4 and CDK6.

Then, the authors looked for a possible action of AMD3100 on several pathways previously demonstrated to be important in GBM cells. They thus observed a decrease (I) of expression and of phosphorylation of the tumor suppressor retinoblastoma $(\mathrm{Rb})$ which is over-activated by mutation or various alterations in $70 \%$ of GBM; (II) of phospho-Akt which is also over-activated in GBM cells both by various tyrosine-kinase receptors (EGFR, PDGFRA, VEGFR2, ...) and by the inactivation of the phosphatase PTEN; (III) of the anti-apoptotic protein BclXL previously shown to be over-expressed in GBM. It is important to note that the effects of AMD3100 on the apoptosis of GBM cells, on their proliferation and on the down-regulation of all these proteins required a long (at least 72 hours) stimulation.

CXCL12 is known to be overexpressed in hypoxic brain or by TGF $\beta$ (12). Calinescu et al. showed that in vitro, $c x c l-12$ is 6 -fold overexpressed with an increase of bif $1-\alpha$ and $\operatorname{tgf}-\beta$ expression, at 96 hours of culture, while the $\operatorname{cxr} 4$ expression remains stable. AMD3100 blocks the increase of expression of $t g f-\beta$ and $c x c l 12$ without any effect on the expression of bif1- $\alpha$. These data suggest that the CXCL12/ CXCR4 pathway operated in GSC cultures as an autocrine positive feedback loop, promoting the survival and the proliferation of these cells.

As their GBM model is artificial (overexpression of SV40lgT and the mutated NRAS in post-natal mouse neural precursors), Calinescu et al. compared at the molecular level the spheres of GBM cells cultivated from their induced GBM and various GBM cells established in cell lines (U251 and U87) or derived from a gliosarcoma (which is a glioma with a high proportion of cells harboring mesenchymal features) and known to be enriched in GSC. Their comparisons were obtained by western blot and concerned some classical GBM markers. The authors conclude that there is a similar molecular profile between their induced-GBM cells and the human cells. However, a deeper molecular analysis, at the transcriptomic or at the proteomic level, would be useful here to assess the model used in the Calinescu's paper.

This deeper molecular analysis between the inducedGBM cells by the SV4LgT and NRAS and the other GBM cells, established as cell lines or derived from tumoral resection, is indeed mandatory as the expression of CXCL12 and CXCR4 but also the effect of a CXCL12- or an AMD3100-stimulation are various regarding other GBM cells tested by the authors.

Finally, Calinescu et al. treated by AMD3100 mice injected at post-natal day 1 with the transforming plasmid. The drug has been delivered by osmotic pumps that have been implanted at day 21 (when tumors develop macroscopically) and left in place for 5 days. The authors observe a non-significant tumor volume decrease when animals receive AMD3100 and a significant decrease of BrdU labeling index in the tumors. However, the median survival of AMD3100-treated animals was significantly higher (53 vs. 30 days) than animals that received saline. These results obtained with a pharmacological approach targeting the CXCR4 signalization were phenocopied using genetic approaches targeting the cxcr4 expression.

The merit of this study is to demonstrate very conclusively the role of the CXCL12/CXCR4 pathway in a model of GBM growth and invasion. However, the first (but also the main) criticism that one can raise is in this interesting paper is about the model. Indeed, the model used by Calinescu et al. has the main advantages to be highly reproducible, to generate operationally-defined GSC and to produce tumors with all the histological characteristics described for GBM. The disadvantages of this model are the cell transformation of postnatal neural precursor, the relationship of the tumor with an immature brain parenchyma and, at least so far, a clear description of the molecular profiling of the tumors both, between themselves and with the human GBM. Indeed, it shouldn't be now a big work to perform RNA sequencing assays of three to 
six of these induced-GBM and to compare with themselves and with data available at the TCGA. This analysis could also be very instructive about the GBM subtype [with or without IDH mutations (13), the classification of those GBM according to Verhaak (14), ...]. Concerning the age of the brain in which the GBM is induced, it could be important as in human patients, GBM in children and GBM in adult are different in their prognosis but also at a molecular level (15).

Finally, one can also have a regret. Indeed, in their model (with all the odds that we have explained), Calinescu et al. clearly demonstrated that the CXCL12/CXCR4 pathway does play a role in tumor growth in vivo as the AMD3100 doubles the mice survival. It would have been interesting to look for a possible role of this signaling pathway in tumor arousal. One has to admit that implanting mini-osmotic pumps in newborn animals is technically difficult. However a targeted gene invalidation approach should be here very informative.

\section{Acknowledgements}

Funding: B Rogister's work is supported by grants from the National Fund for Scientific Research (FNRS/TELEVIE, PDR.15.3621) by the Special Funds of the University of Liège (CFRA.2394) and by a Léon Frédéricq grant.

\section{Footnote}

Conflicts of Interest: The author has no conflicts of interest to declare.

\section{References}

1. Louis DN, Perry A, Reifenberger G, et al. The 2016 World Health Organization Classification of Tumors of the Central Nervous System: a summary. Acta Neuropathol 2016;131:803-20.

2. Ellis HP, Greenslade M, Powell B, et al. Current Challenges in Glioblastoma: Intratumour Heterogeneity, Residual Disease, and Models to Predict Disease Recurrence. Front Oncol 2015;5:251.

3. Oberoi RK, Parrish KE, Sio TT, et al. Strategies to improve delivery of anticancer drugs across the bloodbrain barrier to treat glioblastoma. Neuro Oncol 2016;18:27-36.

4. Lathia JD, Mack SC, Mulkearns-Hubert EE, et al. Cancer stem cells in glioblastoma. Genes Dev 2015;29:1203-17.

5. Calinescu AA, Yadav VN, Carballo E, et al. Survival and Proliferation of Neural Progenitor-Derived Glioblastomas Under Hypoxic Stress is Controlled by a CXCL12/ CXCR4 Autocrine-Positive Feedback Mechanism. Clin Cancer Res 2017;23:1250-62.

6. Piccirillo SG, Spiteri I, Sottoriva A, et al. Contributions to drug resistance in glioblastoma derived from malignant cells in the sub-ependymal zone. Cancer Res 2015;75:194-202.

7. Goffart N, Lombard A, Lallemand F, et al. CXCL12 mediates glioblastoma resistance to radiotherapy in the subventricular zone. Neuro Oncol 2017;19:66-77.

8. Wang R, Chadalavada K, Wilshire J, et al. Glioblastoma stem-like cells give rise to tumour endothelium. Nature 2010;468:829-33.

9. Wiesner SM, Decker SA, Larson JD, et al. De novo induction of genetically engineered brain tumors in mice using plasmid DNA. Cancer Res 2009;69:431-9.

10. Goffart N, Kroonen J, Di Valentin E, et al. Adult mouse subventricular zones stimulate glioblastoma stem cells specific invasion through CXCL12/CXCR4 signaling. Neuro Oncol 2015;17:81-94.

11. Huising MO, Stet RJ, Kruiswijk CP, et al. Molecular evolution of CXC chemokines: extant CXC chemokines originate from the CNS. Trends Immunol 2003;24:307-13.

12. Tabatabai G, Frank B, Möhle R, et al. Irradiation and hypoxia promote homing of haematopoietic progenitor cells towards gliomas by TGF-beta-dependent HIF1alpha-mediated induction of CXCL12. Brain 2006;129:2426-35.

13. Waitkus MS, Diplas BH, Yan H. Isocitrate dehydrogenase mutations in gliomas. Neuro Oncol 2016;18:16-26.

14. Verhaak RG, Hoadley KA, Purdom E, et al. Integrated genomic analysis identifies clinically relevant subtypes of glioblastoma characterized by abnormalities in PDGFRA, IDH1, EGFR, and NF1. Cancer Cell 2010;17:98-110.

15. Aldape K, Zadeh G, Mansouri S, et al. Glioblastoma: pathology, molecular mechanisms and markers. Acta Neuropathol 2015;129:829-48.

Cite this article as: Rogister B. The CXCL12/CXCR4 pathway or the autocrine proliferative loop of the glioblastoma stem cells. Transl Cancer Res 2017. doi: 10.21037/ tcr.2017.03.47 